\title{
THE COMPUTATIONAL COMPLEXITY OF TORSION-FREENESS OF FINITELY PRESENTED GROUPS
}

\author{
STEFFEN LEMPP
}

We determine the complexity of torsion-freeness of finitely presented groups in Kleene's arithmetical hierarchy as $\Pi_{2}^{0}$-complete. This implies in particular that there is no effective listing of all torsion-free finitely presented groups, or of all non-torsion-free finitely presented groups.

\section{INTRODUCTION}

One way of describing a group $G$ is to give its presentation, that is, to write $G$ as

$$
G=\left\langle x_{i}(i \in I) \mid R\right\rangle
$$

(where $\left\{x_{i} \mid i \in I\right\}$ is a set of "generators" and $R$ (the set of "relators") is a set of words in $\left\{x_{i}, x_{i}^{-1} \mid i \in I\right\}$ such that $G \cong F / H$ where $F$ is the free group generated by $\left\{x_{i} \mid i \in I\right\}$ and $H$ is the normal subgroup of $F$ generated by $R$. If we can find a free group $F$ of finite rank and a finite set of relators $R$, then we call $G$ a finitely presented group.

Groups arising in applications, such as fundamental groups in topology, often are given naturally via their presentations. Unfortunately, a finite presentation does not yield very good information about the group. Novikov [6] and Boone [4] showed that in some finitely presented groups, one cannot even tell whether a particular word in $x_{1}, \ldots, x_{n}$ and their inverses is the identity in $G$. (Such groups are said to have unsolvable word problem.) Further work of Baumslag, Boone, and Neumann [3] revealed that many other properties of elements of $G$ also cannot be determined from words denoting the elements.

On the other hand, the finite presentation of a group $G$ also does not allow us to determine almost any conceivable global property of $G$, such as whether $G$ is trivial, finite, Abelian, torsion-free, simple, et cetera. This follows immediately by a theorem

Received 13th November, 1996

This research was partially supported by NSF grant DMS-9504474 and a grant of the British Engineering and Physical Sciences Research Council. The author would like to thank the University of Leeds for their hospitality during his stay there, Reg Allenby (Leeds) for helpful discussions, and Karin Johnsgard (Cornell) for providing a very useful set of lecture notes.

Copyright Clearance Centre, Inc. Serial-fee code: 0004-9729/97 \$A2.00+0.00. 
of Adian [1, 2] and Rabin [7], stating that any Markov property of a finitely presented group cannot be effectively determined from its presentation. (A property of finitely presented groups is called Markov if it holds of some finitely presented group $G_{+}$and fails in any finitely presented group containing some finitely presented group $G_{-}$.)

Once a problem turns out to be undecidable, it is natural to ask exactly how complicated the problem is. Computability (or recursion) theory provides a tool for measuring complexity of unsolvable problems in the form of Kleene's arithmetical hierarchy. For $n>0$, a property (of a "code" $y$ ) is called $\Sigma_{n}^{0}$ if it can be expressed (in the language of arithmetic) by a formula of the form $\exists \vec{x}_{1} \forall \vec{x}_{2} \exists \vec{x}_{3} \ldots Q \vec{x}_{n} R\left(\vec{x}_{1}, \ldots, \vec{x}_{n}, y\right)$ where $R$ contains only bounded quantifiers; it is $\Pi_{n}^{0}$ if it can be expressed by a formula of the form $\forall \vec{x}_{1} \exists \vec{x}_{2} \forall \vec{x}_{3} \ldots Q \vec{x}_{n} R\left(\vec{x}_{1}, \ldots, \vec{x}_{n}, y\right)$. By Post's Theorem, a property is (recursively) enumerable (that is, the set of all objects satisfying the property can be effectively listed) if and only if it is $\Sigma_{1}^{0}$; and a property is decidable (that is, can be determined by an effective algorithm) if and only if it is both $\Sigma_{1}^{0}$ and $\Pi_{1}^{0}$. By a theorem of Kleene, these classes of properties form a proper hierarchy satisfying for all $n>0$ that $\Sigma_{n}^{0}, \Pi_{n}^{0} \subset \Sigma_{n+1}^{0} \cap \Pi_{n+1}^{0} \subset \Sigma_{n+1}^{0}, \Pi_{n+1}^{0}$. A property $P$ is called $\Sigma_{n}^{0}$-hard if any $\Sigma_{n}^{0}$-property $P^{\prime}$ can be effectively reduced to it, that is, there is a computable function $f$ such that, for any (code for a mathematical object) $m, P^{\prime}$ holds of $m$ if and only if $P$ holds of $f(m)$; a property is $\Sigma_{n}^{0}$-complete if it is both $\Sigma_{n}^{0}$ and $\Sigma_{n}^{0}$-complete. Note here that $\Sigma_{n}^{0}$ gives an upper bound on the complexity of a property whereas $\Sigma_{n}^{0}$-hardness gives a lower bound; thus $\Sigma_{n}^{0}$-competeness gives a precise classification of a property in terms of computability and definability. $\left(\Pi_{n}^{0}\right.$-hardness and $\Pi_{n}^{0}$-completeness are defined analogously.)

The above-mentioned Adian-Rabin Theorem actually shows that any Markov property is $\Sigma_{1}^{0}$-hard. Since many Markov properties, such as being trivial, finite, Abelian, et cetera, are also enumerable and so $\Sigma_{1}^{0}$, these properties are thus in fact $\Sigma_{1}^{0}$-complete. Other Markov properties, however, such as being solvable, simple, torsion-free, or having a decidable word problem, are not readily seen to be $\Sigma_{1}^{0}$. One of these, having a decidable word problem, was shown by Boone and Rogers [5] to be $\Sigma_{3}^{0}$-complete.

\section{THE THEOREM}

The main result of this paper is to establish the completeness of the only other Markov property known to be complete at a level other than $\Sigma_{1}^{0}$ :

THEOREM. The property of a finitely presented group being torsion-free is $\Pi_{2}^{0}$ complete. (Thus, of course, the property of a finitely presented group not being torsionfree is $\Sigma_{2}^{0}$-complete.)

This theorem has an immediate consequence about effective enumerations of finitely presented groups: 
COROLlaRY. There are no effective listings of (presentations of) all torsion-free finitely presented groups, or of all non-torsion-free finitely presented groups.

PRoOF: If there were such an effective listing then the set of all finite presentations of such groups would be enumerable, that is, $\Sigma_{1}^{0}$, contradicting the above theorem. $\square$

\section{THE PROOF OF THE THEOREM}

The proof is based on two important theorems of combinatorial group theory, the Higman Embedding Theorem and Britton's Lemma.

We first recall that a group $G=\langle X \mid R\rangle$ is recursively presented if $X$ is a finite set of generators and $R$ is a (recursively) enumerable set of relators; and that $G$ is infinitely recursively presented if $X$ is a countable set of generators and $R$ is a (recursively) enumerable set of relators.

Our proof proceeds by a sequence of reductions, each given by a proposition:

PROPOSITION 1. The property of a recursively presented group being torsionfree can be effectively reduced to the property of a finitely presented group being torsionfree.

PROPOSITION 2. The property of an infinitely recursively presented group being torsion-free can be effectively reduced to the property of a recursively presented group being torsion-free.

PRoposition 3. The property of an infinitely recursively presented group being torsion-free is $\Pi_{2}^{0}$-hard (in fact, $\Pi_{2}^{0}$-complete).

PROOF OF THE THEOREM: The property of a finitely presented group being torsion-free is readily seen to be $\Pi_{2}^{0}$ :

$G$ is torsion-free if and only if $\forall w \forall n>0\left(w^{n} \neq_{G} 1\right.$ or $\left.w=_{G} 1\right)$

where $w$ ranges over words on $X$ and their inverses and $n$ ranges over integers. (Note that equality in $G$, denoted by $=_{G}$, is enumerable and thus $\Sigma_{1}^{0}$.)

But, by Propositions 1, 2, and 3, the property of a finitely presented group being torsion-free is also $\Pi_{2}^{0}$-hard, establishing the theorem.

It now remains to verify the propositions. We first need another definition.

A group $G$ is called an $H N N$-extension (or Britton extension) of a group $H=$ $\langle X \mid S\rangle$ (for a set of generators $X$ and a set of relators $S$ ) if $G=\langle X, t \mid S, R\rangle$ where $t$ is a generator (called a stable letter) not occurring in $X$ and $R$ is a set of relators (that is, words) of the form $a_{i}^{t} b_{i}^{-1}$ (where $i$ ranges over some (possibly infinite, or possibly empty) index set $I$ ) such that the map sending each $a_{i}$ to $b_{i}$ induces an isomorphism of the subgroups of $H$ generated by the $a_{i}$ 's and the $b_{i}$ 's, respectively. (This latter condition is often called the isomorphism condition.) 
Proof OF Proposition 1: Given (a presentation of) a recursively presented group $H=\langle X \mid S\rangle$, we must effectively produce (a presentation of) a finitely presented group $G$ such that $G$ is torsion-free if and only if $H$ is. We note that the Higman Embedding Theorem embeds a recursively presented group $H$ into a finitely presented group $G$. A careful analysis of the proof (for example, in [8]) shows that $G$ is obtained from $H$ by a finite sequence of HNN-extensions. But, by Britton's Lemma, $\mathrm{HNN}$-extensions preserve torsion-freeness, that is, $G$ is torsion-free if and only if $H$ is, as desired.

ProOF OF Proposition 2: Given (a presentation of) an infinitely recursively presented group $H=\langle X \mid S\rangle$, we must effectively produce (a presentation of) a recursively presented group $G$ such that $G$ is torsion-free if and only if $H$ is.

Let $X=\left\{x_{1}, x_{2}, \ldots\right\}$. Let $H^{\prime}$ be the free product $H \star\langle a, b\rangle$ where $\langle a, b\rangle$ is a free group of rank 2. We now form an HNN-extension of $H^{\prime}$ by a stable letter $t$ :

$$
G=\left\langle a, b, x_{1}, x_{2}, \ldots, t \mid R, \boldsymbol{a}^{t} b^{-1}, a^{b^{n} t}\left(x_{n} b^{a^{n}}\right)^{-1}(n>0)\right\rangle
$$

Note here that the isomorphism condition holds since both $\left\{b^{b^{n}} \mid n \geqslant 0\right\}$ and $\{b\} \cup$ $\left\{x_{n} b^{a^{n}} \mid n>0\right\}$ freely generate subgroups in $H^{\prime}$.

But now $G$ is an $\mathrm{HNN}$-extension of $H^{\prime}$, which in turn trivially is an HNN-extension of $H$; so $G$ is torsion-free if and only if $H$ is. Finally, $G$ is finitely generated, namely, by $a$ and $t$.

Proof of Proposition 3: We use the well-known fact (see, for example, [9, p.66]) that the (index) set of all infinite enumerable sets is $\Pi_{2}^{0}$-complete. Given (an index for) an enumerable set $W$, we must thus effectively produce (a presentation of) an infinitely recursively presented group $G$ such that $W$ is infinite if and only if $G$ is torsion-free.

We let $G=\left\langle x_{1}, x_{2}, \cdots \mid R\right\rangle$ where the set of relators is enumerated as follows: Start with $R=\left\{x_{1}^{2}\right\}$ and enumerate the set $W$ stage by stage, enumerating at most one element at any stage. At the stage at which the $n$th element (in the order of enumeration) enters $W$, enumerate into $R$ the set $\left\{x_{n}, x_{n+1}^{2}\right\}$.

Now, if $W$ is finite, say, it contains $n$ elements, then we have

$$
R=\left\{x_{1}^{2}, x_{1}, x_{2}^{2}, x_{2}, \ldots, x_{n}^{2}, x_{n}, x_{n+1}^{2}\right\}
$$

so $G=\left\langle x_{1}, x_{2}, \cdots \mid x_{1}, x_{2}, \ldots, x_{n}, x_{n+1}^{2}\right\rangle \cong\left\langle x_{n+1}, x_{n+2}, \cdots \mid x_{n+1}^{2}\right\rangle$, which has a torsion element $x_{n+1}$ of order 2. On the other hand, if $W$ is infinite, then $R=\left\{x_{1}^{2}, x_{1}, x_{2}^{2}, x_{2}, \ldots\right\}$, so $G=\left\langle x_{1}, x_{2}, \cdots \mid x_{1}, x_{2}, \ldots\right\rangle=1$, which is torsion-free. 


\section{OPEN QuesTIONS}

As mentioned in the introduction, some Markov properties are not known to be (and in fact conjectured not to be) $\Sigma_{1}^{0}$ : Solvability is only known to be $\Sigma_{3}^{0}$, residual finiteness and simplicity are only known to be $\Pi_{2}^{0}$, et cetera. Our technique above, however, does not seem to work for these properties since these are not properties localised at some element and thus not preserved under HNN-extensions.

\section{REFERENCES}

[1] S.I. Adian, 'The unsolvability of certain algorithmic problems in the theory of groups', Trudy Moskov. Mat. Obshch. 6 (1957), 231-298.

[2] S.I. Adian, 'Finitely presented groups and algorithms', Dokl. Akad. Nauk SSSR 117 (1957), 9-12.

[3] G. Baumslag, W.W. Boone and B.H. Neumann, 'Some unsolvable problems about elements and subgroups of groups', Math. Scand. 7 (1959), 191-201.

[4] W.W. Boone, 'Certain simple unsolvable problems in group theory I', Nederl. Akad. Wetensch. Proc. Ser. A 57 (1954), 231-237. 'II', Nederl. Akad. Wetensch. Proc. Ser. A 57 (1954), 492-497. 'III', Nederl. Akad. Wetensch. Proc. Ser. A 58 (1955), 252-256. 'IV', Nederl. Akad. Wetensch. Proc. Ser. A 58 (1955), 571-577. 'V', Nederl. Akad. Wetensch. Proc. Ser. A 60 (1957), 22-27. 'VI', Nederl. Akad. Wetensch. Proc. Ser. A 60 (1957), 227-232.

[5] W.W. Boone and H. Rogers Jr., 'On a problem of J.H.C. Whitehead and a problem of Alonzo Church', Math. Scand. 19 (1966), 185-192.

[6] P.S. Novikov, P. S., 'On the algorithmic unsolvability of the word problem in group theory', Trudy Mat. Inst. Steklov 44 (1955), 1-143.

[7] M.O. Rabin, 'Recursive unsolvability of group theoretic problems', Ann. Math. 67 (1958), $172-194$.

[8] J.J. Rotman, An introduction to the theory of groups (Springer-Verlag, Berlin, Heidelberg, New York, 1995).

[9] R.I. Soare, Recursively enumerable sets and degrees: A study of computable functions and computably generated sets (Springer-Verlag, Berlin, Heidelberg, New York, 1987).

\footnotetext{
Department of Mathematics University of Wisoncsin Madison WI 53706-1388

United States of America

email: lempp@math.wisc.edu
} 